\title{
Lipid Rafts and Bullous Diseases
}

\author{
Elena Zimina ${ }^{1,2}$ and Leena Bruckner-Tuderman ${ }^{*}, 1$ \\ ${ }^{I}$ Department of Dermatology, University Medical Center Freiburg, Freiburg, Germany \\ ${ }^{2}$ University of Toronto, Toronto, Canada
}

\begin{abstract}
Multiple observations point to involvement of lipid membrane domains, known as lipid rafts, in the pathology of human disorders. The putative role of lipid rafts in hereditary and acquired skin blistering diseases is discussed in this review. Stable adhesion of the epidermis to the underlying basement membrane is secured by hemidesmosomes, specialized multiprotein complexes in basal keratinocytes. Loss of function of hemidesmosomal proteins due to inherited or acquired abnormalities result in weak dermal-epidermal adhesion and blistering of the skin. Lipid rafts regulate biological functions of two hemidesmosomal transmembrane components: collagen XVII and $\alpha 6 \beta 4$ integrin. Ectodomain shedding of collagen XVII is regulated by membrane lipid domains, suggesting involvement of lipid rafts in the pathogenesis of junctional epidermolysis bullosa, a genetic disease caused by mutations in the collagen XVII gene, and of bullous pemphigoid, an autoimmune disease with autoantibodies to this collagen. Similarly, adhesive and signaling functions of $\alpha 6 \beta 4$ integrin are modulated by lipid rafts, again linking lipid rafts to junctional epidermolysis bullosa. Therefore, modulation of lipid domains in the epidermis might have therapeutic potential for this group of skin blistering diseases.
\end{abstract}

Keywords: Bullous pemphigoid, collagen XVII, epidermolysis bullosa, hemidesmosome, lipid raft, $\alpha 6 \beta 4$ integrin, skin blistering.

\section{INTRODUCTION}

Increasing evidence points to the role of lipid rafts in a variety of pathologic conditions [1] including Alzheimer's [2] and Parkinson's [3] disease, cardiovascular [4, 5], autoimmune [6,7], infectious [8], inherited [9] and prion disorders. These findings make lipid domains an attractive target for pharmacological trials for treatment and prevention of different diseases. Recent investigations suggest involvement of lipid raft signaling also in the pathogenesis of inflammatory [10] and inherited skin diseases [11, 12], although not very much is known about such events yet. This chapter focuses on what could be the role of lipid rafts in the molecular pathology of skin blistering.

The organization of lipids in the biological membranes can be best explained as mosaic of lipid domains of variable size, so-called lipid rafts [13], highly dynamic cholesterol and sphingolipid enriched domains of plasma membrane, with the size of 10-200 $\mathrm{nm}$. The formation of the isolated lipid domains within the membrane occurs due to different packing capability of sphingolipids and glycosphingolipids (GPL) in the membrane. Sphingolipids contain long saturated acyl chains that allow them to pack together more tightly than unsaturated GPL and thus separate to isolated domains. Cholesterol binds preferentially to sphingolipids and favours the lipid phase separation $[14,15]$. Because of their different density and melting point lipid rafts can float in the more-liquid GPL-rich bulk of the plasma membrane

*Address correspondence to this author at the Department of Dermatology, University Medical Center Freiburg, Hauptstr. 7, D-79104 Freiburg, Germany; Tel: +49 761270 6716; Fax: +49 761270 6936;

E-mail: leena.bruckner-tuderman@uniklinik-freiburg.de and cholesterol can shuttle between the raft and non-raft phase, having the higher affinity to rafts [16]. For more details on the structure and functions of lipid rafts in skin cells, the reader is referred to Chapter 1 .

Importantly, lipid rafts can include or exclude membrane proteins to variable extents. Proteins with raft affinity include GPI-anchored proteins, doubly acylated proteins, cholesterol-linked and palmitoylated proteins, and many transmembrane proteins [17]. The mechanisms of molecular targeting of transmembrane proteins to lipid raft domains is not yet fully understood, but mutational analysis has shown that amino acids in the transmembrane domain near the exoplasmatic leaflet of the membrane are critical for this process [18]. The principle by which rafts exert their functions is a compartmentalisation of specific membrane proteins in the lipid domains. Rafts are dynamic, and both proteins and lipids can move in and out of raft domains with different speed [17]. This variable distribution of proteins between lipid subcompartments could regulate proteinprotein interactions within plasma membrane and affect their functions. Small rafts can contain only a few proteins [17], so to engage in membrane function, they have to cluster together to form the large platform, where the participants in signal transduction process can meet $[19,20]$.

Lipid Rafts Regulate Keratinocyte Functions: Lipid rafts are involved in a broad spectrum of cellular events in epidermal biology. In human keratinocytes, lipid domains were first detected using imaging techniques by labelling with cholera toxin B subunit $[21,22]$, and they have since been shown to play a role in TNF $\alpha-[10]$, EGFR- [23] and cell adhesion associated signaling $[11,12,24,25]$. They also regulate intercellular communication via gap junctions [26] and keratinocyte differentiation [27, 28]. Already these few 
roles indicate the importance of lipid rafts in maintenance of tissue integrity and morphology in the skin, cell growth and survival, modulation of cell behaviour, as well as in inflammatory processes and wound healing. Cholesterol, the major component of lipid rafts, is an important constituent of the cornified layer, as it contributes to epidermal lipid barrier in the skin $[29,30]$. Epidermal differentiation involves a spatially and temporally tightly regulated expression of differentiation-related proteins, and modulation of membrane cholesterol has been shown to alter the expression of epidermal differentiation markers keratin 14 and keratin 10, and to result in the phenotype of early differentiating keratinocytes [28].

Hemidesmosomes Mediate Keratinocyte Adhesion: Adhesion of basal keratinocytes to the underlying basement membrane is secured by hemidesmosomes [31, 32], specialised multiprotein complexes that link keratin cytoskeletal elements through transmembrane components to the extracellular matrix proteins. Hemidesmosome- mediated stable attachment of basal keratinocytes plays a crucial role in maintaining skin integrity. This is indirectly demonstrated by the fact that loss of hemidesmosome function leads to skin blistering in inherited or acquired human diseases and in mouse models.

Two transmembrane proteins, $\alpha 6 \beta 4$ integrin and collagen XVII, play a crucial role in the formation and maintenance of hemidesmosomes. Interaction of $\alpha 6 \beta 4$ integrin with plectin is one of the first steps in the hemidesmosome formation [33] and important for their mechanical stability. $\alpha 6 \beta 4$ integrin appears to be essential for cell adhesion, it connects keratinocytes to one of the major components of the basement membrane, laminin 332 [34]. Collagen XVII belongs to the group of transmembrane collagenous molecules [35] and contributes into maintenance of epidermal-dermal integrity. The intracellular domain of collagen XVII is necessary for the stable attachment of hemidesmosomes to keratin intermediate filaments $[36,37]$, and the extracellular domain of collagen XVII can bind to $\alpha 6$ integrin and laminin 332, and it guides correct integration of laminin 332 into the pericellular matrix [38].

Lipid Rafts and Hemidesmosomal Transmembrane Proteins: $\alpha 6 \beta 4$ integrin localizes to lipid rafts as a result of palmitoylation of the $\beta 4$ subunit, and compartmentalization in lipid rafts potentially explains several aspects of $\alpha 6 \beta 4$ signaling [12]. Palmitoylation-deficient $\alpha 6 \beta 4$ integrin did not associate with rafts or activate SFK (Src family kinase) signaling and failed to promote keratinocyte proliferation in response to EGF, implying that mitogenic signaling requires $\alpha 6 \beta 4$ integrin incorporation into lipid rafts. Furthermore, $\alpha 6 \beta 4$ integrin preferentially binds pSFK in lipid rafts. Palmitoylation is a reversible process, allowing for regulated incorporation of $\alpha 6 \beta 4$ into lipid rafts. Binding to its physiological ligand, laminin 332, or antibody induced oligomerization of $\alpha 6 \beta 4$ increased the amount of integrin recovered in the raft fraction, suggesting that extracellular matrix (ECM) binding of $\alpha 6 \beta 4$ integrin increases its affinity for lipid rafts and enhances signaling. Several other integrins $(\alpha 5 \beta 1 \alpha 6 \beta 1, \alpha 6 \beta 4)$ have also been shown to bind lipid rafts, and functional activation of integrins appears to be linked to the lipid raft localisation [39-41]. One interesting investigation showed that the membrane order itself, i.e. the fluidity of plasma membrane, is highly sensitive to cell-ECM adhesion and to integrin clustering [42]. These data indicate that a significant portion of raft structure is protein dependent, and it is likely that both lipids and proteins cooperate to establish and maintain ordered membrane domains at focal adhesions and to stimulate downstream signaling.

Collagen XVII exists in two forms, as a full length protein and as a soluble ectodomain [43]. Its collagenous ectodomain can be proteolytically released from the cell surface. The cleavage occurs close to the plasma membrane, and is mediated by metalloproteases of the ADAM (A Disintegrin And Metalloprotease). ADAM-17/TACE (TNF-a Converting Enzyme) appears to be a major sheddase of collagen XVII [44]. Collagen XVII localises into lipid domains, and membrane sublocalization of collagen XVII plays a key role in the regulation of its shedding [11]. Distribution of plasma membrane cholesterol strongly affects shedding of collagen XVII, since alteration of cholesterol content increased the proteolytic release of the collagenous ectodomain from the cell surface. TACE is non-raft protease, whereas the majority of collagen XVII molecules are localised in lipid rafts and thus inaccessible for enzymatic processing by TACE (Fig. 1). After disorganisation of the lipid domains collagen XVII molecules become available for TACE, which results in enhanced shedding. Therefore, incorporation of collagen XVII into lipid domains seems to provide an important mechanism to modify accessibility to the sheddases and could regulate interactions of the cell with extracellular ligands. Interestingly, low cholesterol has been shown to promote early keratinocyte differentiation. It seems feasible that this could occur through lipid raft-mediated regulation of collagen XVII shedding, which releases the keratinocytes from their binding partners within the underlying basement membrane. Lipid domains can also regulate ectodomain shedding of other transmembrane collagens, including types XIII [45] and XXIII [46], processed by furin. It seems that lipid domains provide the general mechanism to modulate proteolytic processing of transmembrane collagens.

Genetic Defects of Epidermal Adhesion. Mutations in the genes encoding $\alpha 6 \beta 4$ integrin (ITGA6 and ITGB4) and collagen XVII (COL17A1) are associated with junctional epidermolysis bullosa (EB), an group of genetic skin blistering disorders with variable clinical phenotypes [4749]. Functional consequences of the gene defects include diminished epidermal adhesion and skin blistering in response to minimal trauma or shearing forces. Morphological characteristics of junctional EB are rudimentary hemidesmosomes and subepidermal tissue separation. Clinical hallmarks, in addition to blisters and erosions of the skin and mucous membranes, include nail dystrophy, loss of hair, and dental anomalies (Fig. 2).

Thus far, more than 50 different COL17A1 mutations have been disclosed in patients with junctional EB. Studies on genotype-phenotype correlations have not only revealed the molecular pathomechanisms in this genodermatosis but have augmented our understanding of the normal functions of collagen XVII and its individual subdomains $[35,37,38$, $50,51]$. 


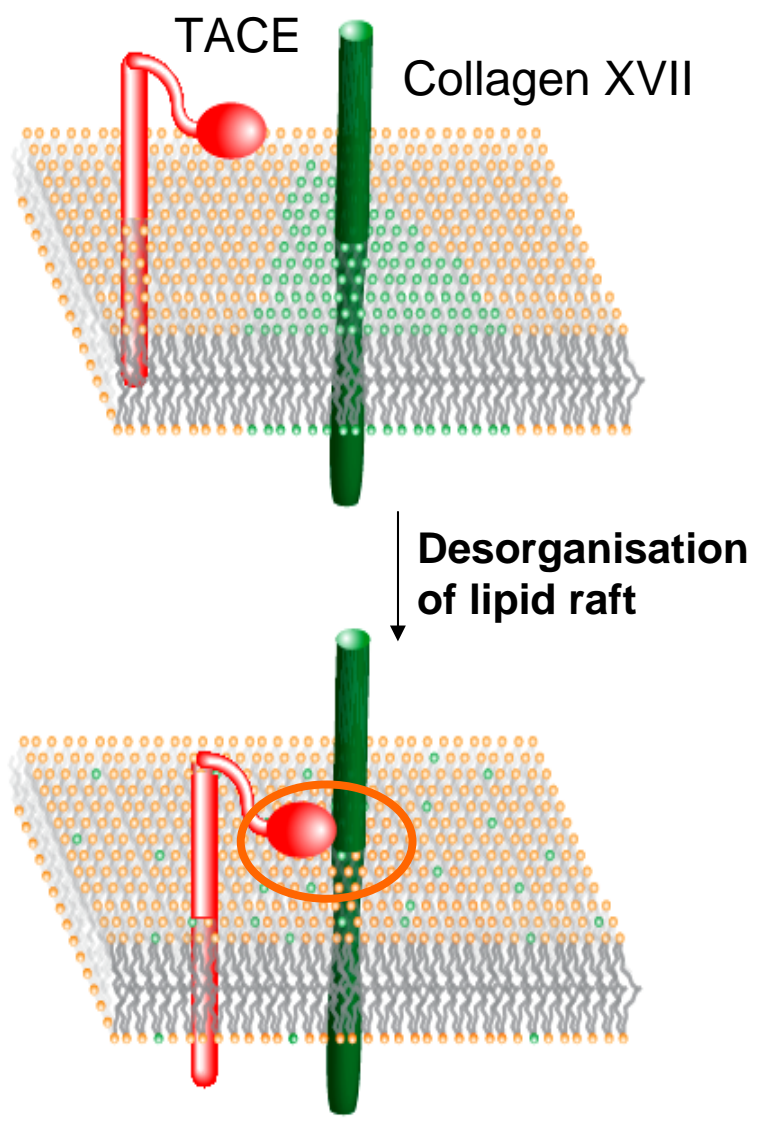

Fig. (1). Integration into lipid rafts regulates shedding of collagen XVII. Collagen XVII mainly associates to lipid rafts, this association provides a key for regulation of its shedding by controlled accessibility to TACE. TACE is not a raft-associated protein, and collagen XVII molecules, mostly in the rafts, are inaccessible for TACE. After disruption of the lipid rafts, collagen XVII molecules become available for enzymatic processing by TACE and shedding is increased.

Ablation or disruption of the genes for the integrin $\alpha 6$ or $\beta 4$ subunits lead to variably severe cases of junctional EB with pyloric atresia. Most mutations target the gene encoding the integrin $\beta 4$ subunit, fewer mutations have been found in the gene for the $\alpha 6$ subunit. A consequence at the ultrastructural level is that hemidesmosomes are absent or hypoplastic. Nonsense mutations result in the absence of the integrin and abolition of all its functions, including binding to laminin 332 and recruitment of plectin to the hemidesmosomal plaque. Missense mutations resulting in amino acid substitution in the plectin-binding region of the integrin $\beta 4$ intracellular domain have been reported to cause milder phenotypes [52].

Significant phenotypic variation of clinical phenotypes in junctional EB still remains poorly understood and has been attributed to genetic modifiers, environmental factors, random events, and interactions between any of these sources [53, 54], but all these are presumptions. In this context there may well be the role of membrane lipids and lipid rafts on the clinical phenotype of EB since both collagen XVII and $\alpha 6 \beta 4$ are lipid raft proteins. As described below, the ligand interactions of collagen XVII and $\alpha 6 \beta 4$ with other proteins or enzymes in the cell membrane may contribute to the pathogenesis of the skin blistering diseases.

Lipid rafts modify epitopes in autoimmune bullous skin diseases. Pemphigoid diseases are a group of acquired autoimmune blistering skin diseases. In bullous pemphigoid (BP), the most frequent autoimmune blistering disorder of the skin, autoantibodies target collagen XVII [55]. The immunodominant epitopes are located within the juxtamembranous NC16a-subdomain [56, 57]. As shown by passive transfer mouse models and in vitro models, antibodies against collagen XVII are pathogenetically relevant, since their binding to the antigen induces complement activation leading to neutrophil infiltration, secretion of proteases and to epidermal-dermal separation [58].

In a subset of BP, the linear IgA dermatosis (LAD), the ectodomain of collagen XVII is specifically targeted by IgAautoantibodies. LAD is characterized by pruritic tense blisters and by tissue-bound and circulating IgAautoantibodies against the epidermal basement membrane zone. In addition to the shed $120 \mathrm{kDa}$ ectodomain of collagen XVII, the IgA-autoantibodies recognize a $97 \mathrm{kDa}$ antigen, which represents a truncated form of the collagen XVII ectodomain.

Lipid rafts have previously been shown to be involved in the pathogenesis of other autoimmune diseases. In systemic lupus erythematosus (SLE), a multiorgan autoimmune
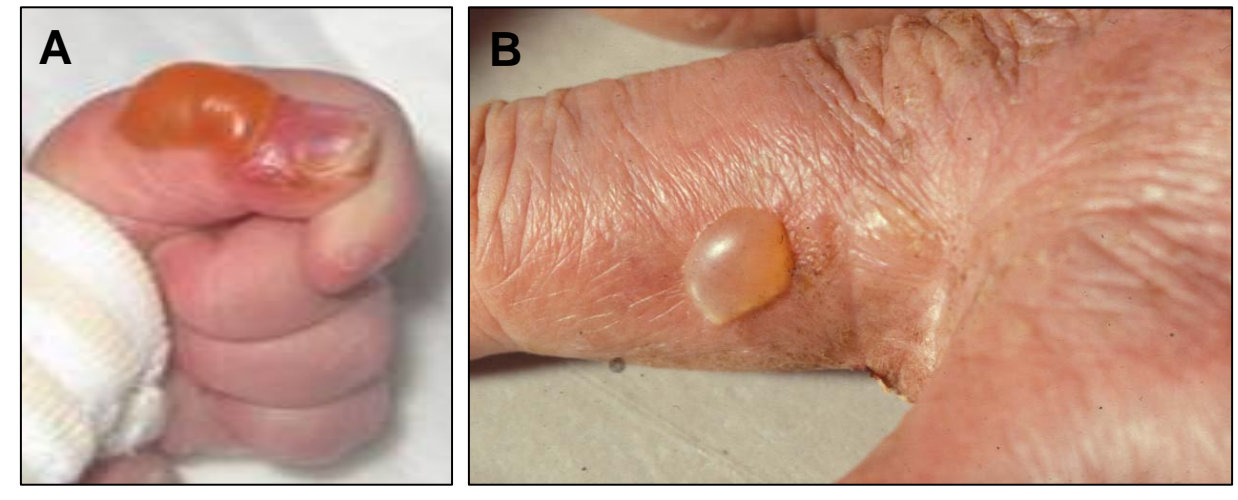

Fig. (2). Skin blistering as a consequence of loss of collagen XVII function in junctional epidermolysis bullosa in an infant (A) and in bullous pemphigoid in an adult (B). 
disease, they can affect $\mathrm{T}$ cells signalling $[6,7]$. Lymphocyte-specific protein tyrosine kinase, critical for maintaining the resting state of $\mathrm{T}$ cells, is mislocalised in SLE T lymphocytes [6]. In normal $\mathrm{T}$ cells, the lymphocytespecific protein tyrosine kinase is mainly localised outside the lipid rafts, but in SLE T cells it associates with the rafts. Another study proved the role of lipid rafts in modulation of higher calcium responses in SLE T cells [7]. A link has been suggested between lipid raft dependent ectodomain shedding of CD30 and certain autoimmune diseases, such as systemic lupus erythematosus, rheumatoid arthritis, systemic sclerosis, atopic dermatitis, Wegener's granulomatosis, Graves' disease, and Hashimoto's thyroiditis, which are associated with increased serum levels of soluble CD30 ectodomain [59].

In both $\mathrm{BP}$ forms, the immunodominant epitopes are localized within the NC-16a subdomain, which harbours the sheddase cleavage site. Both this domain and its proteolytic cleavage seem to play an important pathogenetic role, since shedding of collagen XVII generates neoepitopes, as demonstrated by the fact that the new N-terminus of the shed ectodomain presents a particularly strong epitope for $\mathrm{IgG}$ autoantibodies in BP and $\operatorname{IgA}$ autoantibodies in $\operatorname{LAD}[60$, $61]$.

Thus, all events regulating shedding of collagen XVII, such as lipid rafts or phosphorylation of the cleavage site $[11,62]$, can also influence the pathology in autoimmune blistering skin diseases. It is intriguing to hypothesize that inhibitors of shedding or agents modulating lipid rafts organisation may have therapeutic potential for this group of skin diseases.

\section{ACKNOWLEDGEMENTS}

The authors' work was supported by grant $\mathrm{Br}$ 1475/9-1 from the German Research Foundation DFG and the Epidermolysis bullosa network grant from the German Federal Ministry for Education and Research to LBT.

\section{ABBREVIATIONS}

ADAM $=$ A Disintegrin And Metalloprotease
$\mathrm{BP}=$ Bullous Pemphigoid
$\mathrm{EB}=$ Epidermolysis Bullosa
EGFR $=$ Epidermal Growth Factor Receptor
GPL $=$ Glycosphingolipids
LAD $=$ Linear IgA Dermatosis
SFK $=$ Src Family Kinase
SLE $=$ Systemic Lupus Erythematosus
TACE $=$ TNF- $\alpha$ Converting Enzyme
TNF $\alpha=$ Tumor Necrosis Factor Alpha
REFERENCES

[1] Michel V, Bakovic M. Lipid rafts in health and disease. Biol Cell 2007; 99: 129-40.

[2] Cordy JM, Hooper NM, Turner AJ. The involvement of lipid rafts in Alzheimer's disease. Mol Membr Biol 2006; 23: 111-22.

[3] Fortin DL, Troyer MD, Nakamura K, et al. Lipid rafts mediate the synaptic localization of alpha-synuclein. J Neurosci 2004; 24 : 6715-23.
[4] Li XA, Everson WV, Smart EJ. Caveolae, lipid rafts, and vascular disease. Trends Cardiovasc Med 2005; 15: 92-6.

[5] Callera GE, Montezano AC, Yogi A, et al. Vascular signaling through cholesterol-rich domains: implications in hypertension. Curr Opin Nephrol Hypertens 2007; 16: 90-104.

[6] Jury EC, Kabouridis PS, Flores-Borja F, et al. Altered lipid raftassociated signaling and ganglioside expression in T lymphocytes from patients with systemic lupus erythematosus. J Clin Invest 2004; 113: 1176-87.

[7] Krishnan S, Nambiar MP, Warke VG, et al. Alterations in lipid raft composition and dynamics contribute to abnormal $\mathrm{T}$ cell responses in systemic lupus erythematosus. J Immunol 2004; 172: 7821-31.

[8] Luo C, Wang K, Liu de Q, et al. The functional roles of lipid rafts in $\mathrm{T}$ cell activation, immune diseases and HIV infection and prevention. Cell Mol Immunol 2008; 5: 1-7.

[9] Rakheja D, Narayan SB, Pastor JV, Bennett MJ. CLN3P, the Batten disease protein, localizes to membrane lipid rafts (detergentresistant membranes). Biochem Biophys Res Commun 2004; 317 : 988-91.

[10] Lisby S, Faurschou A, Gniadecki R. The autocrine TNFalpha signalling loop in keratinocytes requires atypical PKC species and NF-kappaB activation but is independent of cholesterol-enriched membrane microdomains. Biochem Pharmacol 2007; 73: 526-33.

[11] Zimina EP, Bruckner-Tuderman L, Franzke CW. Shedding of collagen XVII ectodomain depends on plasma membrane microenvironment. J Biol Chem 2005; 280: 34019-24.

[12] Gagnoux-Palacios L, Dans M, van't Hof W, et al. Compartmentalization of integrin alpha6beta4 signaling in lipid rafts. J Cell Biol 2003; 162: 1189-96.

[13] Jacobson K, Mouritsen OG, Anderson RG. Lipid rafts: at a crossroad between cell biology and physics. Nat Cell Biol 2007; 9: 7-14.

[14] Brown DA, London E. Structure and function of sphingolipid- and cholesterol-rich membrane rafts. J Biol Chem 2000; 275: 17221-4.

[15] Fantini J, Garmy N, Mahfoud R, Yahi N. Lipid rafts: structure, function and role in HIV, Alzheimer's and prion diseases. Expert Rev Mol Med 2002; 4: 1-22.

[16] Simons K, Ikonen E. Functional rafts in cell membranes. Nature 1997; 387: 569-72.

[17] Rajendran L, Simons K. Lipid rafts and membrane dynamics. J Cell Sci 2005; 118: 1099-102.

[18] Scheiffele P, Roth MG, Simons K. Interaction of influenza virus haemagglutinin with sphingolipid-cholesterol membrane domains via its transmembrane domain. EMBO J 1997; 16: 5501-8.

[19] Langlet C, Bernard AM, Drevot P, He HT. Membrane rafts and signaling by the multichain immune recognition receptors. Curr Opin Immunol 2000; 12: 250-5.

[20] Simons K, Ehehalt R. Cholesterol, lipid rafts, and disease. J Clin Invest 2002; 110: 597-603.

[21] Gniadecki R, Bang B. Flotillas of lipid rafts in transit amplifying cell-like keratinocytes. J Invest Dermatol 2003; 121: 522-8.

[22] Gniadecki R, Christoffersen N, Wulf HC. Cholesterol-rich plasma membrane domains (lipid rafts) in keratinocytes: importance in the baseline and UVA-induced generation of reactive oxygen species. J Invest Dermatol 2002; 118: 582-8.

[23] Lambert S, Vind-Kezunovic D, Karvinen S, Gniadecki R. Ligandindependent activation of the EGFR by lipid raft disruption. J Invest Dermatol 2006; 126: 954-62.

[24] Alvares SM, Dunn CA, Brown TA, et al. The role of membrane microdomains in transmembrane signaling through the epithelial glycoprotein Gp140/CDCP1. Biochim Biophys Acta 2008; 1780: 486-96.

[25] Vind-Kezunovic D, Wojewodzka U, Gniadecki R. Focal junctions retard lateral movement and disrupt fluid phase connectivity in the plasma membrane. Biochem Biophys Res Commun 2008; 365: 1-7. Langlois S, Cowan KN, Shao Q, et al. Caveolin-1 and -2 interact with connexin 43 and regulate gap junctional intercellular communication in keratinocytes. Mol Biol Cell 2008; 19: 912-28.

[27] Zheng X, Bollinger Bollag W. Aquaporin 3 colocates with phospholipase $\mathrm{d} 2$ in caveolin-rich membrane microdomains and is downregulated upon keratinocyte differentiation. J Invest Dermatol 2003; 121: 1487-95.

[28] Jans R, Atanasova G, Jadot M, Poumay Y. Cholesterol depletion upregulates involucrin expression in epidermal keratinocytes through activation of p38. J Invest Dermatol 2004; 123: 564-73. 
[29] Menon GK, Feingold KR, Mao-Qiang M, et al. Structural basis for the barrier abnormality following inhibition of HMG CoA reductase in murine epidermis. J Invest Dermatol 1992; 98: 209-19.

[30] Schmidt R, Parish EJ, Dionisius V, et al. Modulation of cellular cholesterol and its effect on cornified envelope formation in cultured human epidermal keratinocytes. J Invest Dermatol 1991; 97: 771-5.

[31] McMillan JR, Akiyama M, Shimizu H. Epidermal basement membrane zone components: ultrastructural distribution and molecular interactions. J Dermatol Sci 2003; 31: 169-77.

[32] Litjens SH, de Pereda JM, Sonnenberg A. Current insights into the formation and breakdown of hemidesmosomes. Trends Cell Biol 2006; 16: 376-83.

[33] Schaapveld RQ, Borradori L, Geerts D, et al. Hemidesmosome formation is initiated by the beta4 integrin subunit, requires complex formation of beta4 and HD1/plectin, and involves a direct interaction between beta4 and the bullous pemphigoid antigen 180 . J Cell Biol 1998; 142: 271-84.

[34] Tsuruta D, Hopkinson SB, Lane KD, et al. Crucial role of the specificity-determining loop of the integrin beta4 subunit in the binding of cells to laminin-5 and outside-in signal transduction. J Biol Chem 2003; 278: 38707-14.

[35] Franzke CW, Tasanen K, Schumann H, Bruckner-Tuderman L. Collagenous transmembrane proteins: collagen XVII as a prototype. Matrix Biol 2003; 22: 299-309.

[36] Borradori L, Chavanas S, Schaapveld RQ, et al. Role of the bullous pemphigoid antigen 180 (BP180) in the assembly of hemidesmosomes and cell adhesion--reexpression of BP180 in generalized atrophic benign epidermolysis bullosa keratinocytes. Exp Cell Res 1998; 239: 463-76.

[37] Huber M, Floeth M, Borradori L, et al. Deletion of the cytoplasmatic domain of BP180/collagen XVII causes a phenotype with predominant features of epidermolysis bullosa simplex. J Invest Dermatol 2002; 118: 185-92.

[38] Tasanen K, Tunggal L, Chometon G, et al. Keratinocytes from patients lacking collagen XVII display a migratory phenotype. Am J Pathol 2004; 164: 2027-38.

[39] Chapman HA, Wei Y, Simon DI, Waltz DA. Role of urokinase receptor and caveolin in regulation of integrin signaling. Thromb Haemost 1999; 82: 291-7.

[40] Decker L, ffrench-Constant C. Lipid rafts and integrin activation regulate oligodendrocyte survival. J Neurosci 2004; 24: 3816-25.

[41] Leitinger B, Hogg N. The involvement of lipid rafts in the regulation of integrin function. J Cell Sci 2002; 115: 963-72.

[42] Gaus K, Le Lay S, Balasubramanian N, Schwartz MA. Integrinmediated adhesion regulates membrane order. J Cell Biol 2006; 174: 725-34.

[43] Schacke H, Schumann H, Hammami-Hauasli N, et al. Two forms of collagen XVII in keratinocytes. A full-length transmembrane protein and a soluble ectodomain. J Biol Chem 1998; 273: 2593743

[44] Franzke CW, Tasanen K, Schacke H, et al. Transmembrane collagen XVII, an epithelial adhesion protein, is shed from the cell surface by ADAMs. Embo J 2002; 21: 5026-35.

[45] Väisänen T, Väisänen MR, Pihlajaniemi T. Modulation of the cellular cholesterol level affects shedding of the type XIII collagen ectodomain. J Biol Chem 2006; 281: 33352-62.
[46] Veit G, Zimina EP, Franzke CW, et al. Shedding of collagen XXIII is mediated by furin and depends on the plasma membrane microenvironment. J Biol Chem 2007; 282: 27424-35.

[47] Pulkkinen L, Uitto J. Hemidesmosomal variants of epidermolysis bullosa. Mutations in the alpha6beta4 integrin and the 180-kD bullous pemphigoid antigen/type XVII collagen genes. Exp Dermatol 1998; 7: 46-64.

[48] Zillikens D, Giudice GJ. BP180/type XVII collagen: its role in acquired and inherited disorders or the dermal-epidermal junction. Arch Dermatol Res 1999; 291: 187-94.

[49] Pulkkinen L, Uitto J. Mutation analysis and molecular genetics of epidermolysis bullosa. Matrix Biol 1999; 18: 29-42.

[50] Nykvist P, Tasanen K, Viitasalo T, et al. The cell adhesion domain of type XVII collagen promotes integrin-mediated cell spreading by a novel mechanism. J Biol Chem 2001; 276: 38673-9.

[51] Vaisanen L, Has C, Franzke C, et al. Molecular mechanisms of junctional epidermolysis bullosa: Col 15 domain mutations decrease the thermal stability of collagen XVII. J Invest Dermatol 2005; 125: 1112-8.

[52] Aumailley M, Has C, Tunggal L, Bruckner-Tuderman L. Molecular basis of inherited skin-blistering disorders, and therapeutic implications. Expert Rev Mol Med 2006; 8: 1-21.

[53] Bruckner-Tuderman L. Secondary modifiers and the phenotypic variability of junctional epidermolysis bullosa. Acta Derm Venereol 2008; 88: 436.

[54] Uitto J. Phenotypic complexity of epidermolysis bullosa: the paradigm of the pruriginosa subtype. Acta Derm Venereol 2009; 89: 4-5.

[55] Sitaru C, Zillikens D. Mechanisms of blister induction by autoantibodies. Exp Dermatol 2005; 14: 861-75

[56] Giudice GJ, Emery DJ, Zelickson BD, et al. Bullous pemphigoid and herpes gestationis autoantibodies recognize a common noncollagenous site on the BP180 ectodomain. J Immunol 1993; 151: 5742-50.

[57] Matsumura K, Amagai M, Nishikawa T, Hashimoto T. The majority of bullous pemphigoid and herpes gestationis serum samples react with the NC16a domain of the $180-\mathrm{kDa}$ bullous pemphigoid antigen. Arch Dermatol Res 1996; 288: 507-9.

[58] Liu Z, Diaz LA, Troy JL, et al. A passive transfer model of the organ-specific autoimmune disease, bullous pemphigoid, using antibodies generated against the hemidesmosomal antigen, BP180. J Clin Invest 1993; 92: 2480-8.

[59] von Tresckow B, Kallen KJ, von Strandmann EP, et al. Depletion of cellular cholesterol and lipid rafts increases shedding of CD30. J Immunol 2004; 172: 4324-31.

[60] Schumann H, Baetge J, Tasanen K, et al. The shed ectodomain of collagen XVII/BP180 is targeted by autoantibodies in different blistering skin diseases. Am J Pathol 2000; 156: 685-95.

[61] Hofmann SC, Voith U, Schonau V, et al. Plasmin Plays a Role in the In Vitro Generation of the Linear IgA Dermatosis Antigen LADB97. J Invest Dermatol 2009.

[62] Zimina EP, Hofmann SC, Fritsch A, et al. Bullous pemphigoid autoantibodies preferentially recognize phosphoepitopes in collagen XVII. J Invest Dermatol 2008; 128: 2736-9.

(c) Zimina and Bruckner-Tuderman; Licensee Bentham Open.

This is an open access article licensed under the terms of the Creative Commons Attribution Non-Commercial License (http: //creativecommons.org/licenses/by-nc/ 3.0/) which permits unrestricted, non-commercial use, distribution and reproduction in any medium, provided the work is properly cited. 